Honam Mathematical J. 33 (2011), No. 4, pp. 499-518

http://dx.doi.org/10.5831/HMJ.2011.33.4.499

\title{
INTERVAL-VALUED FUZZY SUBGROUPS AND HOMOMORPHISMS
}

\author{
HeE Won Kang
}

\begin{abstract}
We obtain the interval-valued fuzzy subgroups generated by interval-valued fuzzy sets and some properties preserved by a ring homomorphism. Furthermore, we introduce the concepts of interval-valued fuzzy coset and study some of it's properties.
\end{abstract}

\section{Introduction}

In 1975, Zadeh[8] introduced the concept of interval-valued fuzzy sets as a generalization of fuzzy sets introduced by himself[7]. After then, Biswas[1] applied the notion of interval-valued fuzzy sets to group theory. Moreover, Gorzalczany[3] applied it to a method of inference in approximate reasoning, and Montal and Samanta[6] applied it to topology. Recently, Hur et al.[4] introduced the concept of an interval-valued fuzzy relations and obtained some of it's properties . Also, Choi et al.[2] applied it to topology in the sense of Šostak, Kang and Hur [5] applied it to algebra.

In this paper, we introduce the notion of interval-valued fuzzy cosets and investigate some of it's properties. Furthermore we obtain the interval-valued fuzzy subgroups generated by interval-valued fuzzy sets and study some properties preserved by a ring homomorphism.

\section{Preliminaries}

We will list some concepts and two results needed in the later sections.

Received August 31, 2011. Accepted September 14, 2011.

2000 Mathematics Subject Classification. 03F55, 20 N25.

Key words and phrases. interval-valued fuzzy subgroup, interval-valued fuzzy coset.

This paper was supported by Woosuk University in 2011. 
Let $D(I)$ be the set of all closed subintervals of the unit interval $I=[0,1]$. The elements of $D(I)$ are generally denoted by capital letters $M, N, \cdots$, and note that $M=\left[M^{L}, M^{U}\right]$, where $M^{L}$ and $M^{U}$ are the lower and the upper end points respectively. Especially, we denoted, $\mathbf{0}$ $=[0,0], \mathbf{1}=[1,1]$, and $\mathbf{a}=[a, a]$ for every $a \in(0,1)$, We also note that

(i) $(\forall M, N \in D(I))\left(M=N \Leftrightarrow M^{L}=N^{L}, M^{U}=N^{U}\right)$,

(ii) $(\forall M, N \in D(I))\left(M \leq N \Leftrightarrow M^{L} \leq N^{L}, M^{U} \leq N^{U}\right)$.

For every $M \in D(I)$, the complement of $M$, denoted by $M^{c}$, is defined by $M^{c}=1-M=\left[1-M^{U}, 1-M^{L}\right](\operatorname{See}[6])$.

Definition 2.1[3,8]. A mapping $A: X \rightarrow D(I)$ is called an intervalvalued fuzzy set (in short, IVFS) in $X$, denoted by $A=\left[A^{L}, A^{U}\right]$, if $A^{L}, A^{U} \in I^{X}$ such that $A^{L} \leq A^{U}$, i.e., $A^{L}(x) \leq A^{U}(x)$ for each $x \in X$, where $A^{L}(x)$ [resp. $A^{U}(x)$ ] is called the lower[resp. upper] end point of $x$ to $A$. For any $[a, b] \in D(I)$, the interval-valued fuzzy set $A$ in $X$ defined by $A(x)=\left[A^{L}(x), A^{U}(x)\right]=[a, b]$ for each $x \in X$ is denoted by $\widetilde{[a, b]}$ and if $a=b$, then the IVFS $[\widetilde{a, b}]$ is denoted by simply $\widetilde{a}$. In particular, $\widetilde{0}$ and $\widetilde{1}$ denote the interval-valued fuzzy empty set and the interval-valued fuzzy whole set in $X$, respectively.

We will denote the set of all IVFSs in $X$ as $D(I)^{X}$. It is clear that set $A=\left[A^{L}, A^{U}\right] \in D(I)^{X}$ for each $A \in I^{X}$.

Definition 2.2[6]. An IVFS A is called an interval-valued fuzzy point(in short, IVFP) in X with the support $x \in X$ and the value $[a, b] \in D(I)$ with $b>0$, denoted by $A=x_{[a, b]}$, if for each $y \in X$,

$$
A(y)= \begin{cases}{[a, b]} & \text { if } y=x \\ \mathbf{0} & \text { otherwise }\end{cases}
$$

In particular, if $b=a$, then $x_{[a, b]}$ is denoted by $x_{\mathbf{a}}$.

We will denote the set of all IVFPs in $X$ as $\operatorname{IVF}_{P}(X)$. 
Definition 2.3[6]. Let $A, B \in D(I)^{X}$ and let $\left\{A_{\alpha}\right\}_{\alpha \in \Gamma} \subset D(I)^{X}$. Then:

(i) $A \subset B$ iff $A^{L} \leq B^{L}$ and $A^{U} \leq B^{U}$.

(ii) $A=B$ iff $A \subset B$ and $B \subset A$.

(iii) $A^{c}=\left[1-A^{U}, 1-A^{L}\right]$.

(iv) $A \cup B=\left[A^{L} \vee B^{L}, A^{U} \vee B^{U}\right]$.

(iv) $\bigcup_{\alpha \in \Gamma} A_{\alpha}=\left[\bigvee_{\alpha \in \Gamma} A_{\alpha}^{L}, \bigvee_{\alpha \in \Gamma} A_{\alpha}^{U}\right]$.

(v) $A \cap B=\left[A^{L} \wedge B^{L}, A^{U} \wedge B^{U}\right]$.

$(\mathrm{v})^{\prime} \bigcap_{\alpha \in \Gamma} A_{\alpha}=\left[\bigwedge_{\alpha \in \Gamma} A_{\alpha}^{L}, \bigwedge_{\alpha \in \Gamma} A_{\alpha}^{U}\right]$.

Result 2.A[6, Theorem 1]. Let $A, B, C \in D(I)^{X}$ and let $\left\{A_{\alpha}\right\}_{\alpha \in \Gamma} \subset$ $D(I)^{X}$. Then:
(a) $\widetilde{0} \subset A \subset \widetilde{1}$.
(b) $A \cup B=B \cup A, A \cap B=B \cap A$.
(c) $A \cup(B \cup C)=(A \cup B) \cup C, A \cap(B \cap C)=(A \cap B) \cap C$.
(d) $A, B \subset A \cup B, A \cap B \subset A, B$.
(e) $A \cap\left(\bigcup_{\alpha \in \Gamma} A_{\alpha}\right)=\bigcup_{\alpha \in \Gamma}\left(A \cap A_{\alpha}\right)$.
(f) $A \cup\left(\bigcap_{\alpha \in \Gamma} A_{\alpha}\right)=\bigcap_{\alpha \in \Gamma}\left(A \cup A_{\alpha}\right)$.
(g) $(\widetilde{0})^{c}=\widetilde{1},(\widetilde{1})^{c}=\widetilde{0}$.
(h) $\left(A^{c}\right)^{c}=A$.
(i) $\left(\bigcup_{\alpha \in \Gamma} A_{\alpha}\right)^{c}=\bigcap_{\alpha \in \Gamma} A_{\alpha}^{c},\left(\bigcap_{\alpha \in \Gamma} A_{\alpha}\right)^{c}=\bigcup_{\alpha \in \Gamma} A_{\alpha}^{c}$.

Definition 2.4[7]. Let $A \in D(I)^{X}$ and let $x_{M} \in \operatorname{IVF}_{P}(X)$. Then:

(i) The set $\left\{x \in X: A^{U}(x)>0\right\}$ is called the support of $A$ and is denoted by $S(A)$.

(ii) $x_{M}$ said to belong to $A$, denoted by $x_{M} \in A$, if $M^{L} \leq A^{L}(x)$ and $M^{U} \leq A^{U}(x)$ for each $x \in X$.

It is obvious that $A=\bigcup_{x_{M} \in A} x_{M}$ and $x_{M} \in A$ if and only if $x_{M^{L}} \in A^{L}$ and $x_{M^{U}} \in A^{U}$. 
Definition 2.5[6]. Let $f: X \rightarrow Y$ be a mapping, let $A=\left[A^{L}, A^{U}\right]$ $\in D(I)^{X}$ and let $B=\left[B^{L}, B^{U}\right] \in D(I)^{Y}$. Then

(a) the image of $A$ under $f$, denoted by $f(A)$, is an IVFS in $Y$ defined as follows: For each $y \in Y$,

$$
f\left(A^{L}\right)(y)= \begin{cases}\bigvee_{y=f(x)} A^{L}(x) & \text { if } f^{-1}(y) \neq \varnothing \\ 0 & \text { otherwise. }\end{cases}
$$

and

$$
f\left(A^{U}\right)(y)= \begin{cases}\bigvee_{y=f(x)} A^{U}(x) & \text { if } f^{-1}(y) \neq \varnothing \\ 0 & \text { otherwise. }\end{cases}
$$

(b) the preimage of $B$ under $f$, denoted by $f^{-1}(B)$, is an IVFS in $Y$ defined as follows: For each $y \in Y$,

$$
f^{-1}\left(B^{L}\right)(y)=\left(B^{L} \circ f\right)(x)=B^{L}(f(x))
$$

and

$$
f^{-1}\left(B^{U}\right)(y)=\left(B^{U} \circ f\right)(x)=B^{U}(f(x))
$$

It can be easily seen that $f(A)=\left[f\left(A^{L}\right), f\left(A^{U}\right)\right]$ and $f^{-1}(B)=$ $\left[f^{-1}\left(B^{L}\right), f^{-1}\left(B^{U}\right)\right]$.

Result 2.B[6, Theorem 2]. Let $f: X \rightarrow Y$ be a mapping and $g: Y \rightarrow Z$ be a mapping. Then
(a) $f^{-1}\left(B^{c}\right)=\left(f^{-1}(B)\right)^{c}, \forall B \in D(I)^{Y}$.
(b) $[f(A)]^{c} \subset f\left(A^{c}\right), \forall A \in D(I)^{Y}$.
(c) $B_{1} \subset B_{2} \Rightarrow f^{-1}\left(B_{1}\right) \subset f^{-1}\left(B_{2}\right)$, where $B_{1}, B_{2} \in D(I)^{Y}$.
(d) $A_{1} \subset A_{2} \Rightarrow f\left(A_{1}\right) \subset f\left(A_{2}\right)$, where $A_{1}, A_{2} \in D(I)^{X}$.
(e) $f\left(f^{-1}(B)\right) \subset B, \forall B \in D(I)^{Y}$.
(f) $A \subset f\left(f^{-1}(A)\right), \forall A \in D(I)^{Y}$.
(g) $(g \circ f)^{-1}(C)=f^{-1}\left(g^{-1}(C)\right), \forall C \in D(I)^{Z}$.
(h) $f^{-1}\left(\bigcup_{\alpha \in \Gamma} B_{\alpha}\right)=\bigcup_{\alpha \in \Gamma} f^{-1} B_{\alpha}$, where $\left\{B_{\alpha}\right\}_{\alpha \in \Gamma} \in D(I)^{Y}$.
(h) $f^{-1}\left(\bigcap_{\alpha \in \Gamma} B_{\alpha}\right)=\bigcap_{\alpha \in \Gamma} f^{-1} B_{\alpha}$, where $\left\{B_{\alpha}\right\}_{\alpha \in \Gamma} \in D(I)^{Y}$.

Definition 2.6[5]. An interval-valued fuzzy set $A$ in $G$ is called an interval-valued fuzzy subgroupoid(in short, IVGP) in $G$ if $A^{L}(x y) \geq A^{L}(x) \wedge A^{L}(y)$ and $A^{U}(x y) \geq A^{U}(x) \wedge A^{U}(y), \forall x, y \in G$.

It is clear that $\widetilde{0}, \widetilde{1} \in \operatorname{IVGP}(G)$. We will denote the IVGPs in $G$ as $\operatorname{IVGP}(G)$. 


\section{Interval-valued fuzzy subgroup generated by an interval- valued fuzzy set}

Definition 3.1[5]. Let $A$ be an IVFS in a set $X$ and let $[\lambda, \mu] \in D(I)$. Then the set $A^{[\lambda, \mu]}=\left\{x \in X: A^{L}(x) \geq \lambda\right.$ and $\left.A^{U}(x) \geq \mu\right\}$ is called a $[\lambda, \mu]$-level subset of $A$.

The following is the immediate result of Definition 3.1.

Proposition 3.2. Let $A$ be an IVFS in a set $X$ and let $\left[\lambda_{1}, \mu_{1}\right],\left[\lambda_{2}, \mu_{2}\right] \in$ $\operatorname{Im}(\mathrm{A})$. If $\lambda_{1}<\lambda_{2}$ and $\mu_{1}<\mu_{2}$, then $A^{\left[\lambda_{1}, \mu_{1}\right]} \supset A^{\left[\lambda_{2}, \mu_{2}\right]}$.

Definition 3.3[5]. Let $G$ be a group and let $A \in D(I)^{G}$. Then $A$ is called an interval-valued fuzzy subgroup (in short, IVG) of $G$ if it satisfies the following conditions :

(i) $A \in \operatorname{IVGP}(\mathrm{G})$, i.e., $A^{L}(x y) \geq A^{L}(x) \wedge A^{L}(y)$ and $A^{U}(x y) \geq$ $A^{U}(x) \wedge A^{U}(y), \forall x, y \in G$.

(ii) $A^{L}\left(x^{-1}\right) \geq A^{L}(x)$ and $A^{U}\left(x^{-1}\right) \geq A^{U}(x), \forall x, y \in G$.

We will denote the set of all IVGs of $G$ as IVG $(G)$.

Result 3.A[1, Proposition 3.1]. Let $A$ be an IVG of a group $G$. Then $A\left(x^{-1}\right)=A(x)$ and $A^{L}(x) \leq A^{L}(e), A^{U}(x) \leq A^{U}(e)$ for each $x \in G$, where $e$ is the identity element of $G$.

Result 3.B[5, Proposition 4.16 and 4.17]. Let $A$ be an IVFS in a group $G$. Then $A \in \operatorname{IVG}(\mathrm{G})$ if and only if $A^{[\lambda, \mu]}$ is a subgroup of $G$ for each $[\lambda, \mu] \in \operatorname{Im}(\mathrm{A})$.

Definition 3.4. Let $A$ be an IVG of a group $G$ and $[\lambda, \mu] \in \operatorname{Im}(\mathrm{A})$. Then the subgroup $A^{[\lambda, \mu]}$ is called a $[\lambda, \mu]$-level subgroup of $A$.

Lemma 3.5. Let $A$ be any IVFS of a set $X$. Then $A^{L}(x)=\bigvee\{\lambda: x \in$ $\left.A^{[\lambda, \mu]}\right\}$ and

$A^{U}(x)=\bigvee\left\{\mu: x \in A^{[\lambda, \mu]}\right\}$, where $x \in X$ and $[\lambda, \mu] \in D(I)$.

Proof. Let $\alpha=\bigvee\left\{\lambda: x \in A^{[\lambda, \mu]}\right\}$, let $\beta=\bigvee\left\{\mu: x \in A^{[\lambda, \mu]}\right\}$ and let $\epsilon>0$ be arbitrary. Then $\alpha-\epsilon<\bigvee\left\{\lambda: x \in A^{[\lambda, \mu]}\right\}$ and 
$\beta-\epsilon<\bigvee\left\{\mu: x \in A^{[\lambda, \mu]}\right\}$. Thus there exist $[\lambda, \mu] \in D(I)$ such that $x \in A^{[\lambda, \mu]}, \alpha-\epsilon<\lambda$ and $\beta-\epsilon<\mu$. Since $x \in A^{[\lambda, \mu]}, A^{L}(x) \geq \lambda$ and $A^{U}(x) \geq \mu$. Thus $A^{L}(x)>\alpha-\epsilon$ and $A^{U}(x)>\beta-\epsilon$. Since $\epsilon>0$ is arbitrary, $A^{L}(x) \geq \alpha$ and $A^{U}(x) \geq \beta$. We now show that $A^{L}(x) \leq \alpha$ and $A^{U}(x) \leq \beta$. Suppose $A^{L}(x)=t_{1}$ and $A^{U}(x)=t_{2}$. Then $\left[t_{1}, t_{2}\right] \in \operatorname{Im}(\mathrm{A})$. Thus $x \in A^{\left[t_{1}, t_{2}\right]}$. So $t_{1} \in\left\{\lambda: x \in A^{[\lambda, \mu]}\right\}$ and $t_{2} \in\left\{\lambda: x \in A^{[\lambda, \mu]}\right\}$. So $t_{1}=\bigvee\left\{\lambda: x \in A^{[\lambda, \mu]}\right\}$ and $t_{2}=\bigvee\left\{\mu: x \in A^{[\lambda, \mu]}\right\}$, i.e. $A^{L}(x) \leq \alpha$ and $A^{U}(x) \leq \beta$. This completes the proof.

We shall denote by $(A)$ the IVG generated by the IVFS $A$ in $G$. We shall use the same notation $\left(A^{[\lambda, \mu]}\right)$ for the ordinary subgroup of the group $G$ generated by the level subset $A^{[\lambda, \mu]}$.

Theorem 3.6. Let $G$ be group and let $A \in D(I)^{G}$. Let $A^{*} \in D(I)^{G}$ be defined as follows: For each $x \in G$,

$\left(A^{*}\right)^{L}(x)=\bigvee\left\{\lambda: x \in\left(A^{[\lambda, \mu]}\right)\right\}$ and $\left(A^{*}\right)^{U}(x)=\bigvee\left\{\mu: x \in\left(A^{[\lambda, \mu]}\right)\right\}$, where $[\lambda, \mu] \in D(I)$. Then $A^{*} \in \operatorname{IVG}(\mathrm{G})$ such that $A^{*}=\bigcap\{B \in$ $\operatorname{IVG}(\mathrm{G}): A \subset B\}$. In this case, $A^{*}$ is called the interval-valued fuzzy subgroup generated by $A$ in $G$ and will be denoted by $(A)$.

Proof. Let $\left[t_{1}, t_{2}\right] \in \operatorname{Im}\left(A^{*}\right)$ and $\alpha=t_{1}-\frac{1}{n}$ and $\alpha=t_{2}-\frac{1}{n}$, where $n$ is

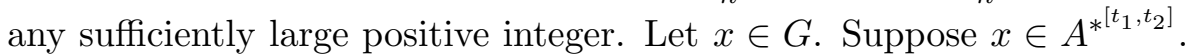
Then $\left(A^{*}\right)^{L}(x) \geq t_{1}$ and $\left(A^{*}\right)^{U}(x) \geq t_{2}$. Thus there exist $[\lambda, \mu] \in D(I)$ such that $\lambda>\alpha, \mu>\beta$ and $x \in A^{[\lambda, \mu]}$. Since $[\alpha, \beta]<[\lambda, \mu]$ and $[\alpha, \beta] \in D(I)$, by Proposition 3.2, $A^{[\lambda, \mu]} \subset A^{[\alpha, \beta]}$. So $x \in A^{[\alpha, \beta]}$, i.e., $x \in\left(A^{[\alpha, \beta]}\right)$. Now suppose $x \in\left(A^{[\lambda, \mu]}\right)$. Then $\alpha \in\left\{\lambda: x \in\left(A^{[\lambda, \mu]}\right)\right\}$ and $\beta \in\left\{\mu: x \in\left(A^{[\lambda, \mu]}\right)\right\}$. Thus $\alpha \leq \bigvee\left\{\lambda: x \in\left(A^{[\lambda, \mu]}\right)\right\}$ and $\beta \leq \bigvee\{\mu:$ $\left.x \in\left(A^{[\lambda, \mu]}\right)\right\}$. So $t_{1}-\frac{1}{n} \leq\left(A^{*}\right)^{L}(x)$ and $t_{2}-\frac{1}{n} \leq\left(A^{*}\right)^{U}(x)$, i.e., $t_{1} \leq$ $\left(A^{*}\right)^{L}(x)$ and $t_{2} \leq\left(A^{*}\right)^{U}(x)$. Hence $x \in A^{\left.* t_{1}, t_{2}\right]}$, i.e., $\left(A^{*[\alpha, \beta]}\right) \subset A^{*^{\left[t_{1}, t_{2}\right]}}$. Therefore $A^{*\left[t_{1}, t_{2}\right]}=\left(A^{*[\alpha, \beta]}\right)$. Since $\left(A^{*[\alpha, \beta]}\right)$ is a subgroup of $G, A^{*\left[t_{1}, t_{2}\right]}$ is a subgroup of $G$. By Result 3.B, $A^{*} \in \operatorname{IVG}(\mathrm{G})$.

Now, we show that $A \subset A^{*}$. Let $x \in G$. Then, by Lemma 3.5, $\left(A^{*}\right)^{L}(x)=\bigvee\left\{\lambda: x \in A^{[\lambda, \mu]}\right\}$ and $\left(A^{*}\right)^{U}(x)=\bigvee\left\{\mu: x \in A^{[\lambda, \mu]}\right\}$. Thus $\left(A^{*}\right)^{L}(x) \leq \bigvee\left\{\lambda: x \in\left(A^{[\lambda, \mu]}\right)\right\}$ and $\left(A^{*}\right)^{U}(x) \leq \bigvee\left\{\mu: x \in\left(A^{[\lambda, \mu]}\right)\right\}$. So $A \subset A^{*}$. Finally, let $B$ be any IVG of $G$ such that $A \subset B$. We show that $A^{*} \subset B$. Let $x \in G$ and $A^{*}(x)=\left[t_{1}, t_{2}\right]$. Then $A^{*^{\left[t_{1}, t_{2}\right]}}=\left(A^{[\alpha, \beta]}\right)$, where $\alpha=t_{1}-\frac{1}{n}, \beta=t_{2}-\frac{1}{n}$, and $n$ is any sufficiently large positive integer. Thus $x \in\left(A^{[\alpha, \beta]}\right)$. So $x=a_{1} a_{2} \cdots a_{m}$, where $a_{i}$ or $a_{i}^{-1}$ belongs to $A^{[\alpha, \beta]}(i=1, \cdots, m)$. 
On the other hand,

$$
\begin{aligned}
B^{L}(x) & =B^{L}\left(a_{1} a_{2} \cdots a_{m}\right) \\
& \geq B^{L}\left(a_{1}\right) \wedge B^{L}\left(a_{2}\right) \wedge \cdots \wedge B^{L}\left(a_{m}\right) \\
& \geq A^{L}\left(a_{1}\right) \wedge A^{L}\left(a_{2}\right) \wedge \cdots \wedge A^{L}\left(a_{m}\right) \\
& \geq \alpha=t_{1}-\frac{1}{n} .
\end{aligned}
$$

By the similar arguments, we have that $B^{U}(x) \geq \beta=t_{2}-\frac{1}{n}$. Since $n$ is sufficiently large positive integer, $B^{L}(x) \geq t_{1}$ and $B^{U}(x) \geq t_{2}$. So $A^{*} \subset B$. Hence $A^{*}=\bigcap\{B \in \operatorname{IVG}(\mathrm{G}): A \subset B\}$. This completes the proof.

It is possible that card $\operatorname{Im}\left(A^{*}\right)$ be less than card $\operatorname{Im}(A)$. Moreover, $\operatorname{Im}\left(A^{*}\right)$ need not be contained in $\operatorname{Im}(A)$ as shown in the following examples.

Example 3.7. let $G=\{e, a, b, c\}$ be the Klein four -group, where $a^{2}=b^{2}=e$ and $a b=b a$. Define an IVFS $A$ of $G$ by: $A(e)=$ $[0.5,0.5], A(a)=[0.2,0.8], A(b)=[0.3,0.7], A(a b)=[0.4,0.6]$. Then $A^{[0.2,0.8]}=\{a\}, A^{[0.3,0.7]}=\{a, b\}, A^{[0.4,0.6]}=\{a, b, a b\}$ and $A^{[0.5,0.5]}=G$. Thus $\left(A^{[0.2,0.8]}\right)=\{e, a\}$ and $\left(A^{[0.3,0.7]}\right)=G$. Moreover, by definition, we have $A^{*}(e)=A^{*}(a)=[0.2,0.8]$ and $A^{*}(b)=A^{*}(a b)=[0.3,0.7]$.

Now an attempt is made to obtain a necessary and sufficient condition for a p-group to be cyclic.

Lemma 3.8. Let $G$ be a finite group. Suppose there exists $A \in \operatorname{IVG}(\mathrm{G})$ satisfying the following conditions: For any $x, y \in G$,

(i) $A(x)=A(y) \Rightarrow(x)=(y)$.

(ii) $A^{L}(x)>A^{L}(y)$ and $A^{U}(x)>A^{U}(y) \Rightarrow(x) \subset(y)$.

Then $G$ is cyclic.

Proof. Suppose $A$ is constant on $G$. Then $A(x)=A(y)$ for any $x, y \in$ $G$. By the condition (i), $(x)=(y)$. So $G=(x)$. Now suppose $A$ is not constant on $G$. Let $\operatorname{Im}(A)=\left\{\left[t_{0}, s_{0}\right],\left[t_{1}, s_{1}\right], \cdots,\left[t_{n}, s_{n}\right]\right\}$, where $t_{0}>t_{1}>\cdots>t_{n}$ and $s_{0}>s_{1}>\cdots>s_{n}$. Then, by Proposition 3.2 and Result 3.B, we obtain the chain of level subgroups of $A$ :

$A^{\left[t_{0}, s_{0}\right]} \subset A^{\left[t_{1}, s_{1}\right]} \subset \cdots \subset A^{\left[t_{n}, s_{n}\right]}=G$.

Let $x \in G-A^{\left[t_{n-1}, s_{n-1}\right]}$. We show that $G=(x)$. Let $g \in G-A^{\left[t_{n-1}, s_{n-1}\right]}$. Since $t_{0}>t_{1}>\cdots>t_{n}$ and $s_{0}>s_{1}>\cdots>s_{n}, A(g)=A(x)=$ 
$A^{\left[t_{n-1}, s_{n-1}\right]}$. By the condition (i), $(g)=(x)$. Thus $G-A^{\left[t_{n-1}, s_{n-1}\right]} \subset(x)$. Now let $g \in A^{\left[t_{n-1}, s_{n-1}\right]}$. Then $A^{L}(g) \geq t_{n-1}>t_{n}=A^{L}(x)$ and $A^{U}(g) \geq s_{n-1}>s_{n}=A^{U}(x)$. By the condition (ii), $(g)=(x)$. Thus $A^{\left[t_{n-1}, s_{n-1}\right]} \subset(x)$. So $G=(x)$. Hence, in either cases, $G$ is cyclic.

Lemma 3.9. Let $G$ be a cyclic group of order $p^{n}$, where $p$ is prime. Then there exists $A \in \operatorname{IVG}(\mathrm{G})$ satisfying the following conditions: For any $x, y \in G$,

(i) $A(x)=A(y) \Rightarrow(x)=(y)$.

(ii) $A^{L}(x)>A^{L}(y)$ and $A^{U}(x)>A^{U}(y) \Rightarrow(x) \subset(y)$.

Proof. Consider the following chain of subgroups of $G$ :

$(e)=G_{0} \subset G_{1} \subset \cdots \subset G_{n-1} \subset G_{n}=G$,

where $G_{i}$ is the subgroup of $G$ generated by an element of order $p^{i}$, $i=0,1, \cdots, n$ and $e$ is the identity of $G$. We define a mapping $A: G \rightarrow$ $D(I)$ as follows: For each $x \in G, A(e)=\left[t_{0}, s_{0}\right]$ and $A(x)=\left[t_{i}, s_{i}\right]$ if $x \in G_{i}-G_{i-1}$ for any $i=1,2, \cdots, n$, where $\left[t_{i}, s_{i}\right] \in D(I)$ such that $t_{0}>t_{1}>\cdots>t_{n}$ and $s_{0}>s_{1}>\cdots>s_{n}$. Then we can easily check that $A \in \operatorname{IVG}(\mathrm{G})$ satisfying the conditions (i) and (ii).

From Lemmas 3.8 and 3.9, we obtain the following.

Theorem 3.10. Let $G$ be a group of order $p^{n}$. Then $G$ is cyclic if and only if there exists $A \in \operatorname{IVG}(\mathrm{G})$ satisfying the following conditions: For any $x, y \in G$,

(i) $A(x)=A(y) \Rightarrow(x)=(y)$.

(ii) $A^{L}(x)>A^{L}(y)$ and $A^{U}(x)>A^{U}(y) \Rightarrow(x) \subset(y)$.

\section{Interval-valued fuzzy ideals and homomorphisms}

Definition 4.1[5]. Let $(R,+, \cdot)$ be a ring and let $\tilde{0} \neq A \in D(I)^{R}$. Then $A$ is called an interval- valued fuzzy subring (in short, $I V R$ ) in $R$ if it satisfies the following conditions:

(i) $A$ is an IVG in $R$ with respect to the operation "+" (in the sense of Definition 3.3).

(ii) $A$ is an IVGP in $R$ with respect to the operation ". " (in the sense of Definition 2.6). 
It is clear that subrings of $R$ are IVRs of $R$. We will denote the set of all IVRs of $R$ as $\operatorname{IVR}(\mathrm{R})$.

Definition 4.2[5]. Let $R$ be a ring and let $\tilde{0} \neq A \in D(I)^{R}$. Then $A$ is called an interval- valued fuzzy ideal (in short, IVI) of $R$ if it satisfies the following conditions:

(i) $A$ is an IVR of $R$.

(ii) $A^{L}(x y) \geq A^{L}(x), A^{U}(x y) \geq A^{U}(x)$ and $A^{L}(x y) \geq A^{L}(y), A^{U}(x y) \geq$ $A^{U}(y)$ for any $x, y \in R$.

We will denote the set of all IVIs of $R$ as IVI(R).

Result 4.A[5, Proposition 6.5]. Let $R$ be a ring and let $\tilde{0} \neq A \in$ $D(I)^{R}$. Then $A \in \operatorname{IVR}(R)$ if and only if for any $x, y \in R$,

(i) $A^{L}(x-y) \geq A^{L}(x) \wedge A^{L}(y)$ and $A^{U}(x-y) \geq A^{U}(x) \wedge A^{U}(y)$.

(ii) $A^{L}(x y) \geq A^{L}(x) \wedge A^{L}(y)$ and $A^{U}(x y) \geq A^{U}(x) \wedge A^{U}(y)$.

It is clear that if $A$ is an $\operatorname{IVI}(\mathrm{R})$, then $A(-x)=A(x) \leq A(0)$ for each $x \in R$, where 0 is the identity in $\mathrm{R}$ with respect to " + ".

Proposition 4.3. Let $A$ be an IVFS in a ring R. Then $A \in \mathrm{IVI}(\mathrm{R})$ if and only if $A^{[\lambda, \mu]}$ is an ideal of $\mathrm{R}$ for each $[\lambda, \mu] \in \operatorname{Im}(\mathrm{A})$.

Proof. $(\Rightarrow)$ : Suppose $A \in \operatorname{IVI}(\mathrm{R})$. For each $[\lambda, \mu] \in \operatorname{Im}(A)$, let $x, y \in$ $A^{[\lambda, \mu]}$. Then $A^{L}(x) \geq \lambda, A^{U}(x) \geq \mu$ and $A^{L}(y) \geq \lambda, A^{U}(y) \geq \mu$. By Result 4.A (i), $A^{L}(x-y) \geq A^{L}(x) \wedge A^{L}(y)$ and $A^{U}(x-y) \geq A^{U}(x) \wedge$ $A^{U}(y)$. Thus $A^{L}(x-y) \geq \lambda$ and $A^{U}(x-y) \geq \mu$. So $x-y \in A^{[\lambda, \mu]}$. Let $x \in R$ and $y \in A^{[\lambda, \mu]}$. Then $A^{L}(y) \geq \lambda$ and $A^{U}(y) \geq \mu$. Since $A \in \mathrm{IVI}(\mathrm{R})$, by Result 4 .A (ii), $A^{L}(x y) \geq A^{L}(x) \wedge A^{L}(y)$ and $A^{U}(x y) \geq$ $A^{U}(x) \wedge A^{U}(y)$. Thus $A^{L}(x y) \geq \lambda$ and $A^{U}(x y) \geq \mu$. So $x y \in A^{[\lambda, \mu]}$. Similarly, we have $y x \in A^{[\lambda, \mu]}$. Hence $A^{[\lambda, \mu]}$ is an ideal of $\mathrm{R}$.

$(\Leftarrow)$ : Suppose the necessary holds. For any $x, y \in R$, let $A(x)=$ $\left[t_{1}, s_{1}\right]$ and $A(y)=\left[t_{2}, s_{2}\right]$. Then clearly $x \in A^{\left[t_{1}, s_{1}\right]}$ and $y \in A^{\left[t_{2}, s_{2}\right]}$. Since $A^{\left[t_{1}, s_{1}\right]}$ is an ideal of $R, x-y \in A^{\left[t_{1}, s_{1}\right]}$. Then $A^{L}(x-y) \geq t_{1} \geq$ $t_{1} \wedge t_{2}=A^{L}(x) \wedge A^{L}(y)$ and $A^{U}(x-y) \geq s_{1} \geq s_{1} \wedge s_{2}=A^{U}(x) \wedge A^{U}(y)$. Thus $A$ satisfies the condition (i) of Result 4.A. Now for each $x \in R$, let $A(x)=[\lambda, \mu]$. Then clearly $x \in A^{[\lambda, \mu]}$. Let $y \in R$. Since $A^{[\lambda, \mu]}$ is an ideal of $R, x y \in A^{[\lambda, \mu]} y x \in A^{[\lambda, \mu]}$. Then $A^{L}(x y) \geq \lambda=A^{L}(x), A^{U}(x y) \geq \mu=$ $A^{U}(x)$ and $A^{L}(y x) \geq \lambda=A^{L}(y), A^{U}(y x) \geq \mu=A^{U}(y)$. Thus $A$ satisfies 
the condition (ii) of Definition 4.2. Hence $A$ is an IVI of $R$.

Example 4.4. Let $R$ denote the ring of real numbers under the usual operations of addition and multiplication. We define a mapping $A: R \rightarrow$ $D(I)$ as follows: For each $x \in R$,

$A(x)= \begin{cases}{[t, s]} & \text { if } \mathrm{x} \text { is rational, } \\ {\left[t^{\prime}, s^{\prime}\right]} & \text { if } \mathrm{x} \text { is irrational }\end{cases}$

where $[t, s],\left[t^{\prime}, s^{\prime}\right] \in D(I)$ such that $t>t^{\prime}$ and $s>s^{\prime}$. Then we can see that $A \in \operatorname{IVR}(\mathrm{R})$ but $A \notin \mathrm{IVI}(\mathrm{R})$.

Definition 4.5[5]. Let $X$ and $Y$ be sets, let $f: X \rightarrow Y$ be a mapping and let $A \in D(I)^{X}$. Then $A$ is said to be interval-valued fuzzy invariant(in short, IVF-invariant) if $f(x)=f(y)$ implies $A(x)=A(y)$, i.e., $A^{L}(x)=A^{L}(y)$ and $A^{U}(x)=A^{U}(y)$.

It is clear that if $A$ is IVF-invariant, then $f^{-1}(f(A))=A$.

Definition 4.6[5]. Let $(X, \circ)$ be a groupoid and let $A, B \in D(I)^{X}$. Then the interval-valued fuzzy product of $A$ and $B, A \circ B$, is defined as follow : For each $x \in X$,

$$
(A \circ B)^{L}(x)= \begin{cases}\bigvee_{(y, z) \in X \times X}\left(A^{L}(y) \wedge B^{L}(z)\right) & \text { if } \mathrm{x}=\mathrm{yz}, \\ 0 & \text { otherwise }\end{cases}
$$

and

$$
(A \circ B)^{U}(x)= \begin{cases}\bigvee_{(y, z) \in X \times X}\left(A^{U}(y) \wedge B^{U}(z)\right) & \text { if } \mathrm{x}=\mathrm{yz}, \\ 0 & \text { otherwise. }\end{cases}
$$

Similarly, we have the following definition.

Definition 4.7. Let $A$ and $B$ be any two IVIs of a ring $R$. Then the interval-valued fuzzy sum of $A$ and $B, A+B$, is defined as follow : For each $x \in X$,

$$
(A+B)^{L}(x)= \begin{cases}\bigvee_{(y, z) \in X \times X}\left(A^{L}(y) \wedge B^{L}(z)\right) & \text { if } \mathrm{x}=\mathrm{y}+\mathrm{z}, \\ 0 & \text { otherwise }\end{cases}
$$

and 


$$
(A+B)^{U}(x)= \begin{cases}\bigvee_{(y, z) \in X \times X}\left(A^{U}(y) \wedge B^{U}(z)\right) & \text { if } \mathrm{x}=\mathrm{y}+\mathrm{z} \\ 0 & \text { otherwise }\end{cases}
$$

This section reflects the effect of a homomorphism on the sum, product and intersection of any two IVIs of a ring.

Proposition 4.8. Let $f: R \rightarrow R^{\prime}$ be a ring epimorphism. If $A$ and $B$ are IVIs of $R$, then
(a) $f(A+B)=f(A)+f(B)$,
(b) $f(A \circ B)=f(A) \circ f(B)$,
(c) $f(A \cap B)=f(A) \cap f(B)$, with equality if at least one of $A$ or $B$ is IVF-invariant.

Proof. (a) Let $y \in R^{\prime}$ and let $\epsilon>0$ be arbitrary. Let $\left[\alpha, \alpha^{\prime}\right]=f(A+$ $B)(y)$ and let $\left[\beta, \beta^{\prime}\right]=(f(A)+f(B))(y)$.

Then

$$
\begin{aligned}
\alpha & =f(A+B)^{L}(y)=\bigvee_{z \in f^{-1}(y)}(A+B)^{L}(z), \\
\alpha^{\prime} & =f(A+B)^{U}(y)=\bigvee_{z \in f^{-1}(y)}(A+B)^{U}(z)
\end{aligned}
$$

and

$$
\begin{aligned}
& \beta=(f(A)+f(B))^{L}(y)=\bigvee_{y=z+z^{\prime}}\left(f(A)^{L}(z) \wedge f(B)^{L}\left(z^{\prime}\right)\right) \\
& \beta^{\prime}=(f(A)+f(B))^{U}(y)=\bigvee_{y=z+z^{\prime}}\left(f(A)^{U}(z) \wedge f(B)^{U}\left(z^{\prime}\right)\right)
\end{aligned}
$$

Thus $\alpha-\epsilon<\bigvee_{z \in f^{-1}(y)}(A+B)^{L}(z)$ and $\alpha^{\prime}-\epsilon<\bigvee_{z \in f^{-1}(y)}(A+B)^{U}(z)$. So there exist $z_{0}, z_{0}^{\prime} \in R$ with $f\left(z_{0}\right)=y$ and $f\left(z_{0}^{\prime}\right)=y$ such that $\alpha-\epsilon<(A+B)^{L}\left(z_{0}\right)$ and $\alpha-\epsilon<(A+B)^{U}\left(z_{0}^{\prime}\right)$. By the definition of sum,

$\alpha-\epsilon<\bigvee_{z_{0}=a+b}\left(A^{L}(a) \wedge B^{L}(b)\right)$ and $\alpha^{\prime}-\epsilon<\bigvee_{z_{0}^{\prime}=a^{\prime}+b^{\prime}}\left(A^{L}\left(a^{\prime}\right) \wedge\right.$ $\left.B^{L}\left(b^{\prime}\right)\right)$.

Then there exist $a_{0}, b_{0} \in R$ with $z_{0}=a_{0}+b_{0}$ such that $\alpha-\epsilon<\left(A^{L}\left(a_{0}\right) \wedge\right.$ $\left.B^{L}\left(b_{0}\right)\right)$ and there exist $a_{0}^{\prime}, b_{0}^{\prime} \in R$ with $z_{0}^{\prime}=a_{0}^{\prime}+b_{0}^{\prime}$ such that $\alpha^{\prime}-\epsilon<$ $\left(A^{U}\left(a_{0}^{\prime}\right) \wedge B^{U}\left(b_{0}^{\prime}\right)\right)$. 
On the other hand,

$$
\begin{aligned}
\beta & \geq f(A)^{L}\left(f\left(a_{0}\right)\right) \wedge f(B)^{L}\left(f\left(b_{0}\right)\right) \\
& =f\left(A^{L}\right)\left(f\left(a_{0}\right)\right) \wedge f\left(B^{L}\right)\left(f\left(b_{0}\right)\right) \\
& =f^{-1}\left(f\left(A^{L}\right)\right)\left(a_{0}\right) \wedge f^{-1}\left(f\left(B^{L}\right)\right)\left(b_{0}\right) \\
& \geq A^{L}\left(a_{0}\right) \wedge B^{L}\left(b_{0}\right) .
\end{aligned}
$$

Similarly, we have $\beta^{\prime} \geq A^{U}\left(a_{0}^{\prime}\right) \wedge B^{U}\left(b_{0}^{\prime}\right)$. So $\beta>\alpha-\epsilon$ and $\beta^{\prime}>\alpha^{\prime}-\epsilon$. Since $\epsilon$ is arbitrary, $\beta \geq \alpha$ and $\beta^{\prime} \geq \alpha^{\prime}$. Hence

$$
[f(A)+f(B)]^{L}(y) \geq f(A+B)^{L}(y) \text { for each } y \in R^{\prime} . \text { (4.1) }
$$

Now we will show that $\beta \leq \alpha$ and $\beta^{\prime} \leq \alpha^{\prime}$. Clearly,

and

$$
\beta-\epsilon<\bigvee_{y=z+z^{\prime}}\left(f(A)^{\bar{L}}(z) \wedge f(B)^{\bar{L}}\left(z^{\prime}\right)\right)
$$

$$
\beta-\epsilon<\bigvee_{y=z+z^{\prime}}\left(f(A)^{U}(z) \wedge f(B)^{U}\left(z^{\prime}\right)\right) .
$$

Then there exist $z_{1}, z_{1}^{\prime} \in R^{\prime}$ with $y=z_{1}+z_{1}^{\prime}$ such that

and

$$
\beta-\epsilon<f(A)^{L}\left(z_{1}\right)=\bigvee_{x \in f^{-1}\left(z_{1}\right)} A^{L}(x)
$$

$$
\beta-\epsilon<f(B)^{L}\left(z_{1}\right)=\bigvee_{x \in f^{-1}\left(z_{1}^{\prime}\right)} A^{L}(x) .
$$

Hence there exist $z_{0}, z_{0}^{\prime} \in R^{\prime}$ with $y=z_{0}+z_{0}^{\prime}$ such that

and

$$
\beta-\epsilon<f(A)^{U}\left(z_{0}\right)=\bigwedge_{x \in f^{-1}\left(z_{0}\right)} A^{U}(x)
$$

$$
\beta-\epsilon<f(B)^{U}\left(z_{0}^{\prime}\right)=\bigwedge_{x \in f^{-1}\left(z_{0}^{\prime}\right)} B^{U}(x) .
$$

Thus there exist $x_{1}, x_{1}^{\prime} \in R$ with $f\left(x_{1}\right)=z_{1}, f\left(x_{1}^{\prime}\right)=z_{1}^{\prime}$ such that

and

$$
\beta-\epsilon<A^{L}\left(x_{1}\right), \beta-\epsilon<B^{L}\left(x_{1}^{\prime}\right)
$$

there exist $x_{0}, x_{0}^{\prime} \in R$ with $f\left(x_{0}\right)=z_{0}, f\left(x_{0}^{\prime}\right)=z_{0}^{\prime}$ such that $\beta-\epsilon<f^{U}\left(x_{0}\right), \beta-\epsilon<B^{U}\left(x_{0}^{\prime}\right)$. So

$$
\begin{aligned}
\beta-\epsilon & <A^{L}\left(x_{1}\right) \wedge B^{L}\left(x_{1}^{\prime}\right) \leq(A+B)^{L}\left(x_{1}+x_{1}^{\prime}\right) \\
& \leq \bigvee_{x \in f^{-1}(y)}(A+B)^{L}(x)=f(A+B)^{L}(y)
\end{aligned}
$$

and

$$
\begin{aligned}
\beta^{\prime}-\epsilon & <A^{U}\left(x_{0}\right) \wedge B^{U}\left(x_{0}^{\prime}\right) \leq(A+B)^{U}\left(x_{0}+x_{0}^{\prime}\right) \\
& \leq \bigvee_{x \in f^{-1}(y)}(A+B)^{U}(x)=f(A+B)^{U}(y) .
\end{aligned}
$$


Hence $\beta-\epsilon<\alpha$ and $\beta^{\prime}-\epsilon<\alpha^{\prime}$. Since $\epsilon>0$ is arbitrary, $\beta \leq \alpha$ and $\beta^{\prime} \leq \alpha^{\prime}$. So

$$
(f(A)+f(B))(y) \leq f(A+B)(y) \text { for each } y \in R^{\prime} .(4.2)
$$

Therefore, by (4.1) and (4.2), $f(A)+f(B)=f(A+B)$.

(b) Let $y \in R^{\prime}$ and let $\epsilon>0$ be arbitrary. Let $\left[\alpha, \alpha^{\prime}\right]=f(A \circ B)(y)$ and $\left[\beta, \beta^{\prime}\right]=(f(A) \circ f(B))(y)$. Then

$$
\begin{aligned}
\alpha & =f(A \circ B)^{L}(y)=\bigvee_{x \in f^{-1}(y)}(A \circ B)^{L}(z), \\
\alpha^{\prime} & =f(A \circ B)^{U}(y)=\bigvee_{x \in f^{-1}(y)}(A \circ B)^{U}(z)
\end{aligned}
$$

and

$$
\begin{aligned}
& \beta=(f(A) \circ f(B))^{L}(y)=\bigvee_{y=y_{1} y_{2}}\left(f(A)^{L}\left(y_{1}\right) \wedge f(B)^{L}\left(y_{2}\right)\right) \\
& \beta^{\prime}=(f(A) \circ f(B))^{U}(y)=\bigvee_{y=y_{1} y_{2}}\left(f(A)^{U}\left(y_{1}\right) \wedge f(B)^{U}\left(y_{2}\right)\right)
\end{aligned}
$$

In (4.3), $\alpha-\epsilon<\bigvee_{z \in f^{-1}(y)}(A \circ B)^{L}(z)$ and $\alpha^{\prime}-\epsilon<\bigvee_{z \in f^{-1}(y)}(A \circ$ $B)^{U}(z)$. Thus there exist $x, x^{\prime} \in f^{-1}(y)$ such that $\alpha-\epsilon<(A \circ B)^{L}(x)$ and $\alpha^{\prime}-\epsilon<(A \circ B)^{U}(x)$. Since $(A \circ B)^{L}(x)=\bigvee_{x=x_{1} x_{2}}\left(A^{L}\left(x_{1}\right) \wedge B^{L}\left(x_{2}\right)\right)$ and $(A \circ B)^{U}\left(x^{\prime}\right)=\bigvee_{x^{\prime}=x_{1}^{\prime} x_{2}^{\prime}}\left(A^{U}\left(x_{1}^{\prime}\right) \wedge B^{U}\left(x_{2}^{\prime}\right)\right)$, there exist $x_{1}, x_{2}, x_{1}^{\prime}, x_{2}^{\prime} \in R$ with $x=x_{1} x_{2}$ and $x^{\prime}=x_{1}^{\prime} x_{2}^{\prime}$ such that $\alpha-\epsilon<A^{L}\left(x_{1}\right) \wedge B^{L}\left(x_{2}\right)$ and $\alpha^{\prime}-\epsilon<A^{U}\left(x_{1}^{\prime}\right) \wedge B^{U}\left(x_{2}^{\prime}\right)$. Since $A \subset f^{-1}(f(A))$, by Result 2.B(f), $A^{L} \leq f^{-1}(f(A))^{L}$ and $A^{U} \leq f^{-1}(f(A))^{U}$. On the other hand,

$$
f^{-1}(f(A))^{L}=f^{-1}\left(f(A)^{L}\right)=f^{-1}\left(f\left(A^{L}\right)\right)
$$

and

$$
\begin{aligned}
& f^{-1}(f(A))^{U}=f^{-1}\left(f(A)^{U}\right)=f^{-1}\left(f\left(A^{U}\right)\right) . \text { Thus } \\
& \alpha-\epsilon<f^{-1}\left(f(A)^{L}\right)\left(x_{1}\right) \wedge f^{-1}\left(f(B)^{L}\right)\left(x_{2}\right) \\
&=f(A)^{L}\left(f\left(x_{1}\right) \wedge f(B)^{L}\left(f\left(x_{2}\right)\right)\right. \\
& \leq \bigvee_{y=y_{1} y_{2}}\left(f(A)^{L}\left(y_{1}\right) \wedge f(B)^{L}\left(y_{2}\right)\right) \\
&=(f(A) \circ f(B))^{L}(y)=\beta .
\end{aligned}
$$


By the similar arguments, we have that $\alpha^{\prime}-\epsilon \leq(f(A) \circ f(B))^{U}(y)=\beta^{\prime}$. Since $\epsilon>0$ is arbitrary, $\alpha \leq \beta$ and $\alpha^{\prime} \leq \beta^{\prime}$. In (4.4),

$$
\begin{aligned}
\beta-\epsilon & <\bigvee_{y=y_{1} y_{2}}\left(f(A)^{L}\left(y_{1}\right) \wedge f(B)^{L}\left(y_{2}\right)\right)\left(y_{2}\right) \\
& =\bigvee_{y=y_{1} y_{2}}\left(\left(\bigvee_{z_{1} \in f^{-1}\left(y_{1}\right)} A^{L}\left(z_{1}\right)\right) \wedge\left(\bigvee_{z_{2} \in f^{-1}\left(y_{2}\right)} B^{L}\left(z_{2}\right)\right)\right)
\end{aligned}
$$

and

$$
\begin{aligned}
\beta^{\prime}-\epsilon & <\bigvee_{y=y_{1} y_{2}}\left(f(A)^{U}\left(y_{1}\right) \wedge f(B)^{U}\left(y_{2}\right)\right) \\
& =\bigvee_{y=y_{1} y_{2}}\left(\left(\bigvee_{z_{1} \in f^{-1}\left(y_{1}\right)} A^{U}\left(z_{1}\right)\right) \wedge\left(\bigvee_{z_{2} \in f^{-1}\left(y_{2}\right)} B^{U}\left(z_{2}\right)\right)\right) .
\end{aligned}
$$

Thus there exist $y_{1}, y_{2} \in R^{\prime}$ with $y=y_{1} y_{2}$ such that

$$
\begin{aligned}
\beta-\epsilon & <\left(\bigvee_{z_{1} \in f^{-1}\left(y_{1}\right)} A^{L}\left(z_{1}\right)\right) \wedge\left(\bigvee_{z_{2} \in f^{-1}\left(y_{2}\right)} B^{L}\left(z_{2}\right)\right) \\
& =\bigvee_{z_{1} \in f^{-1}\left(y_{1}\right)} \bigvee_{z_{2} \in f^{-1}\left(y_{2}\right)}\left(A^{L}\left(z_{1}\right) \wedge B^{L}\left(z_{2}\right)\right)
\end{aligned}
$$

and

$$
\begin{aligned}
\beta^{\prime}-\epsilon & <\left(\bigvee_{z_{1} \in f^{-1}\left(y_{1}\right)} A^{U}\left(z_{1}\right)\right) \wedge\left(\bigvee_{z_{2} \in f^{-1}\left(y_{2}\right)} B^{U}\left(z_{2}\right)\right) \\
& =\bigvee_{z_{1} \in f^{-1}\left(y_{1}\right)} \bigvee_{z_{2} \in f^{-1}\left(y_{2}\right)}\left(A^{U}\left(z_{1}\right) \wedge B^{U}\left(z_{2}\right)\right) .
\end{aligned}
$$

So there exist $x_{1} \in f^{-1}\left(y_{1}\right)$ and $x_{2} \in f^{-1}\left(y_{2}\right)$ such that $\beta-\epsilon<A^{L}\left(x_{1}\right) \wedge$ $B^{L}\left(x_{2}\right)$ and $\beta-\epsilon<A^{U}\left(x_{1}\right) \wedge B^{U}\left(x_{2}\right)$.

Let $x=x_{1} x_{2}$. Since $f$ is a ring homomorphism, $y=y_{1} y_{2}=f\left(x_{1} x_{2}\right)=$ $f(x)$. Thus

$$
\begin{aligned}
A^{L}\left(x_{1}\right) \wedge B^{L}\left(x_{2}\right) & \leq \bigvee_{x=x_{1} x_{2}}\left(A^{L}\left(x_{1}\right) \wedge B^{L}\left(x_{2}\right)\right) \\
& =(A \circ B)^{L} \leq \bigvee_{x \in f^{-1}(y)}(A \circ B)^{L}(x) \\
& =f(A \circ B)^{L}(y)=\alpha
\end{aligned}
$$

By the similar arguments, we have that $A^{U}\left(x_{1}\right) \wedge B^{U}\left(x_{2}\right) \leq f(A \circ$ $B)^{U}(y)=\alpha^{\prime}$. So $\beta-\epsilon<\alpha$ and $\beta^{\prime}-\epsilon<\alpha^{\prime}$. Since $\epsilon>0$ is arbitrary, $\beta \leq \alpha$ and $\beta^{\prime} \leq \alpha^{\prime}$. Hence $[\alpha, \beta]=\left[\alpha^{\prime}, \beta^{\prime}\right]$. Therefore $f(A \circ B)=f(A) \circ f(B)$. 
(c) Clearly, $A \cap B \subset A$ and $A \cap B \subset B$. By Result 2.B(d), $f(A \cap B) \subset$ $f(B)$. So $f(A \cap B) \subset f(A) \cap f(B)$. Suppose $B$ is IVF-invariant. Then clearly, $f^{-1}(f(B))=B$. Let $y \in R^{\prime}$ and let $\epsilon>0$ is arbitrary. Let $[\alpha, \beta]=(f(A) \cap f(B))(y)$ and let $\left[\alpha^{\prime}, \beta^{\prime}\right]=(f(A) \cap f(B))(y)$. Then and

$$
\alpha=(f(A) \cap f(B))^{L}(y)=\left(\bigvee_{x \in f^{-1}(y)} A^{L}(x)\right) \wedge f(B)^{L}(y)
$$

$$
\beta=(f(A) \cap f(B))^{U}(y)=\left(\bigvee_{x \in f^{-1}(y)} A^{U}(x)\right) \wedge f(B)^{U}(y) .
$$

Thus $\alpha-\epsilon<\left(\bigvee_{x \in f^{-1}(y)} A^{L}(x)\right) \wedge f(B)^{L}(y)$ and $\beta-\epsilon<\left(\bigvee_{x \in f^{-1}(y)} A^{U}(x)\right) \wedge$ $f(B)^{U}(y)$. So there exists an $x \in f^{-1}(y)$ such that

$$
\alpha-\epsilon<A^{L}(x), \alpha-\epsilon<f(B)^{L}(y)
$$

and

$$
\beta-\epsilon<A^{U}(x), \alpha-\epsilon<f(B)^{U}(y) .
$$

Since $B$ is IVF-invariant, $f^{-1}(f(B))=B$. Then $B^{L}(x)$

$$
f(B)^{L}(y)=f(B)^{L}(f(x))=f^{-1}\left(f(B)^{L}\right)(x)=f^{-1}\left(f\left(B^{L}\right)\right)(x)=
$$

and $B^{U}(x)$.

$f(B)^{U}(y)=f(B)^{U}(f(x))=f^{-1}\left(f(B)^{U}\right)(x)=f^{-1}\left(f\left(B^{U}\right)\right)(x)=$

Thus $\alpha-\epsilon<A^{L}(x), \alpha-\epsilon<B^{L}(x)$ and $\beta-\epsilon<A^{U}(x), \beta-\epsilon<B^{U}(x)$. So $\alpha-\epsilon<A^{L}(x) \wedge B^{L}(x)=(A \cap B)^{L}(x)$ and $\beta-\epsilon<A^{U}(x) \wedge B^{U}(x)=$ $(A \cap B)^{L}(x)$.

Hence

and

$$
\alpha-\epsilon<\bigvee_{x \in f^{-1}(y)}(A \cap B)^{L}(x)=\left(f(A \cap B)^{L}\right)(y)=\alpha^{\prime}
$$

$\alpha-\epsilon<\bigvee_{x \in f^{-1}(y)}(A \cap B)^{U}(x)=\left(f(A \cap B)^{U}\right)(y)=\beta^{\prime}$. Since $\epsilon>0$ is arbitrary, $\alpha \leq \alpha^{\prime}$ and $\beta \leq \beta^{\prime}$. Thus $f(A) \cap f(B) \subset f(A \cap B)$. Therefore $f(A) \cap f(B)=f(A \cap B)$.

\section{Interval-valued fuzzy cosets}

Definition 5.1. Let $A$ be any IVI of a ring $R$ and let $x \in R$. Then $A_{x} \in D(I)^{R}$ is called the interval-valued fuzzy coset determined by $x$ and $A$ if $A_{x}(r)=A(r-x)$ for each $r \in R$.

Proposition 5.2. Let $R$ be any IVI of a ring $R$ and let $R / A$ the set of all interval-valued fuzzy cosets of $A$ in $R$. Then $R / A$ is a ring under the following operations:

$$
A_{x}+A_{y}=A_{x+y} \text { and } A_{x} A_{y}=A_{x y} \text { for any } x, y \in R .
$$


Proof. For any $a, b, c, d \in R$, suppose $A_{a}=A_{b}$ and $A_{c}=A_{d}$. Then $A(r-a)=A(r-b)$ for each $r \in R(5.1)$

and

$A(r-c)=A(r-d)$ for each $r \in R .(5.2)$

Let $r=a+c-d$ in (5.1), $r=c$ in (5.2) and $r=a$ in (5.1). Then

$A(a+c-d-a)=A(a+c-d-b)=A(c-d)$,

$A(c-c)=A(c-d)=A(0)(5.3)$

and

$$
\begin{aligned}
A(a-a)=A(a-b) & =A(0) . \text { (5.4) On the other hand, } \\
\left(A_{a}+A_{c}\right)^{L}(r) & =A_{a+c}^{L}(r)=A^{L}(r-a-c) \\
& =A^{L}((r-b-d)-(a+c-b-d)) \\
& \geq A^{L}(r-b-d) \wedge A^{L}(a+c-b-d) \\
& =A^{L}(r-b-d) \wedge A^{L}(0)(\operatorname{By}(5.3)) \\
& =A^{L}(r-b-d) \\
& =A_{b+d}^{L}(r)=\left(A_{b}+A_{d}\right)^{L}(r) .
\end{aligned}
$$

By the similar arguments, we have that $\left(A_{a}+A_{c}\right)^{U}(r)=\left(A_{b}+A_{d}\right)^{U}(r)$. Thus $A_{a}+A_{d} \subset A_{a}+A_{c}$. Similarly, we have $A_{a}+A_{c} \subset A_{b}+A_{d}$. So $A_{a}+A_{c}=A_{b}+A_{d}$. Hence addition is well-defined. Also,

$$
\begin{aligned}
\left(A_{a} A_{c}\right)^{L}(r) & =A_{a c}^{L}(r)=A^{L}(r-a c) \\
& =A^{L}((r-b d)-(a c-b d)) \\
& \geq A^{L}(r-b d) \wedge A^{L}(a c-b d) \\
& =A^{L}(r-b d) \wedge A^{L}((a-b) c-b(d-c))(\text { By (5.3) and (5.4)) } \\
& \left.\geq A^{L}(r-b d) \wedge A^{L}(a-b) A^{L}(d-c)\right) \\
& =A^{L}(r-b d) \wedge A^{L}(0) A^{L}(0)(\operatorname{By}(5.4) \text { and (5.5)) } \\
& =A^{L}(r-b d)=A_{b d}^{L}(r)=A_{b}^{L} A_{d}^{L}(r) .
\end{aligned}
$$

By the similar arguments, we have that $\left(A_{a} A_{c}\right)^{U}(r)=\left(A_{b} A_{d}\right)^{U}(r)$. Thus $A_{b} A_{d} \subset A_{a} A_{c}$. Similarly, we have $A_{a} A_{c} \subset A_{b} A_{d}$. So $A_{b} A_{d}=A_{a} A_{c}$. Hence multiplication is well-defined. Clearly, $A_{0}(=A)$ acts as the additive identity, $A_{e}$ as the multiplicative identity (where $e$ is the multiplicative identity of $R$ ) and $A_{-x}$ as additive inverse of $A_{x}$. It is now a purely routine matter to verify the other properties. This completes the proof. 
Lemma 5.3. Let $A$ be any IVR or an IVI of a ring $R$. If there exist $x, y \in R$ such that $A^{L}(x)<A^{L}(y)$ and $A^{U}(x)<A^{U}(y)$, then $A(x-y)=A(x)=A(y-x)$.

Proof. Since $A$ is an IVG of $R$ with respect to "+", by Result 4.A, $A(x-y)=A(y-x)$. Thus it is sufficient to show that $A(x-y)=A(x)$. Since $A^{L}(x)<A^{L}(y), A^{U}(x)<A^{U}(y)$ and $A$ is an IVR or an IVI of $R$, $A^{L}(x-y) \geq A^{L}(x) \wedge A^{L}(y)=A^{L}(x)$ and $A^{U}(x-y) \geq A^{U}(x) \wedge A^{U}(y)=$ $A^{U}(x)$. On the other hand, $A^{L}(x)=A^{L}(x-y+y) \geq A^{L}(x-y) \wedge A^{L}(y)$ and $A^{U}(x)=A^{U}(x-y+y) \geq A^{U}(x-y) \wedge A^{U}(y)$. Thus $A^{L}(x) \geq A^{L}(x-y)$ and $A^{U}(x) \geq A^{U}(x-y)$. So $A^{L}(x-y)=A(x)$. This completes the proof.

Lemma 5.4. If $A$ is any IVI of a ring $R$, then $A(x)=A(0)$ if and only if $A_{x}=A_{0}$, where $x \in R$.

Proof. $(\Rightarrow)$ : Suppose $A(x)=A(0)$. Since $A$ is an IVG of $R$ with respect to "+", $A(r) \leq A(0)=A(r)$, i.e., $A^{L}(r) \leq A^{L}(0)=A^{L}(r)$ and $A^{U}(r) \leq A^{U}(0)=A^{U}(r)$ for each $r \in R$.

Case (i): Suppose $A(r)<A(x)$. Then, by Lemma 5.3, $A(r-x)=$ $A(x)$. Thus $A_{x}(r)=A_{0}(r)$ for each $r \in R$.

Case (ii): Suppose $A(r)=A(x)$. Then $x, r \in A^{[\lambda, \mu]}$, where $[\lambda, \mu]=$ $A(0)$. Since $A$ is an IVG of $R, A^{[\lambda, \mu]}$ is a subgroup of $R$. Thus $x-r \in$ $A^{[\lambda, \mu]}$. Thus $A^{L}(x-r) \leq \lambda=A^{L}(0)$ and $A^{U}(x-r) \geq \mu=A^{U}(0)$. Since $A^{L}(x-r) \leq A^{L}(0)$ and $A^{U}(x-r) \leq A^{U}(0), A^{L}(x-r)=A^{L}(0)$ and $A^{U}(x-r)=A^{U}(0)$. Thus $A(x-r)=A(0)=A(x)=A(r)$, i.e., $A_{x}(r)=A_{0}(r)$ for each $r \in R$. In either case, $A_{x}(r)=A_{0}(r)$ for each $r \in R$. Hence $A_{x}=A_{0}$ for each $r \in R$.

$(\Leftarrow)$ : It is straightforward.

Proposition 5.5. Let $A$ be any IVI of a ring $R$ and let $A(0)=[\lambda, \mu]$. Then $R / A^{[\lambda, \mu]} \cong R / A$.

Proof. Define a mapping $f: R \rightarrow R / A$ by $f(x)=A_{x}$ for each $r \in R$. Then it is easy to check that $f$ is a ring epimorphism. By Lemma 5.4,

$$
\begin{aligned}
\text { Kerf } & =\left\{x \in R: f(x)=A_{0}\right\}=\left\{x \in R: A_{x}=A_{0}\right\} \\
& =\left\{x \in R: A(x)=A_{0}\right\}=A^{[\lambda, \mu]} .
\end{aligned}
$$

Hence $R / A^{[\lambda, \mu]} \cong R / A$. 
Proposition 5.6. Let $f: R \rightarrow R^{\prime}$ be a ring epimorphism and let $A$ be an IVI of $R$ such that $A^{[\lambda, \mu]} \subset \operatorname{Kerf}$, where $[\lambda, \mu]=A(0)$. Then there exists a unique epimorphism $\bar{f}: R / A \rightarrow R^{\prime}$ such that $f=\bar{f} \circ g$, where $g(x)=A_{x}$ for each $r \in R$.

Proof. Define a mapping $\bar{f}: R_{A} \rightarrow R^{\prime}$ by $\bar{f}\left(A_{x}\right)=f(x)$ for each $r \in R$. Suppose $A_{x}=A_{y}$. Then $A_{x-y}=A_{0}=A_{x}=A_{y}$. By Lemma 5.4, $A(x-y)=A(x)$. Then $x-y \in A^{[\lambda, \mu]}$. Since $A^{[\lambda, \mu]} \subset K \operatorname{Kerf}$, $x-y \in \operatorname{Kerf}$. Thus $f(x)=f(y)$, i.e., $\bar{f}\left(A_{x}\right)=\bar{f}\left(A_{y}\right)$. So $\bar{f}$ is welldefined. Furthermore, since $f$ is surjective, $\bar{f}$ is also surjective. Moreover, it is easy to see that $\bar{f}$ is a homomorphism.

Consider the following diagram:

$R$

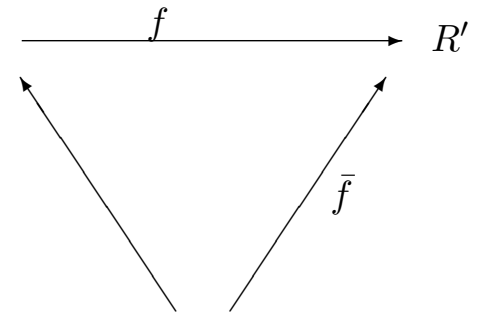

$$
R / A
$$

Let $x \in R$. Then $f(x)=\bar{f}\left(A_{x}\right)=\bar{f}(g(x))=(\bar{f} \circ g)(x)$. Thus the above diagram commutes, i.e., $f=\bar{f} \circ g$.

Suppose there exists an epimorphism $h: R / A \rightarrow R^{\prime}$ such that $f=$ $h \circ g$. Let $x \in R$. Then $\bar{f}\left(A_{x}\right)=f(x)=(h \circ g)(x)=h(g(x))=h\left(A_{x}\right)$. Thus $\bar{f}=h$. So $\bar{f}$ is unique. This completes the proof.

Corollary 5.6. The induced homomorphism $\bar{f}$ is an isomorphism if and only if $A$ is IVF-invariant.

Proof. $(\Rightarrow)$ : Suppose $\bar{f}$ is an isomorphism, i.e., $\bar{f}$ is injective. For any $x, y \in R$, let $f(x)=f(y)$. Then $\bar{f}\left(A_{x}\right)=\bar{f}\left(A_{y}\right)$. Since $\bar{f}$ is injective, $A_{x}=A_{y}$. Thus $A_{x-y}=A_{0}$. By Lemma 5.4, $A(x-y)=A(0)$. By Proposition 4.7 in [5], $A(x)=A(y)$. So $A$ is IVF-invariant.

$(\Leftarrow)$ : Suppose $A$ is IVF-invariant and $\bar{f}\left(A_{x}\right)=\bar{f}\left(A_{y}\right)$. Then $f(x)=$ $f(0)$. Since $A$ is IVF-invariant, $A(x)=A(0)$. By Lemma 5.4, $A_{x}=A_{0}$. So $\bar{f}$ is injective. This completes the proof. 
Proposition 5.7. Let $f: R \rightarrow R^{\prime}$ be a ring epimorphism and let $A$ be an IVF-invariant IVI of $R$. Then $R / A=R^{\prime} / f(A)$.

Proof. Since $A$ is IVF-invariant, $\operatorname{Kerf} \subset A^{[\lambda, \mu]}$, where $[\lambda, \mu]=A(0)$. Consider $f(A)\left(0^{\prime}\right)=\left[f\left(A^{L}\right)\left(0^{\prime}\right), f\left(A^{U}\right)\left(0^{\prime}\right)\right]$, where $0^{\prime}$ denotes the additive identity in $R^{\prime}$. Then

$$
f\left(A^{L}\right)\left(0^{\prime}\right)=\bigvee_{x \in f^{-1}\left(0^{\prime}\right)} A^{L}(x) \text { and } f\left(A^{U}\right)\left(0^{\prime}\right)=\bigvee_{x \in f^{-1}\left(0^{\prime}\right)} A^{U}(x)
$$

Since $f(0)=0^{\prime}$ and $A(x) \leq A(0)$, i.e., $A^{L}(x) \leq A^{L}(0), A^{U}(x) \leq A^{U}(0)$ for each $x \in R, A^{L}(x)=A^{L}(0)$ and $A^{U}(x)=A^{U}(0)$, i.e., $f(A)\left(0^{\prime}\right)=$ $A(0)=[\lambda, \mu]$. Now,

$$
\begin{aligned}
f(x) \in[f(A)]^{[\lambda, \mu]} & \Leftrightarrow f(A)^{L}(f(X)) \geq \lambda \text { and } f(A)^{U}(f(X)) \geq \mu \\
& \Leftrightarrow f\left(A^{L}\right)(f(x)) \geq \lambda \text { and } f\left(A^{U}\right)(f(x)) \geq \mu \\
& \Leftrightarrow f^{-1}\left(f\left(A^{L}\right)\right)(x) \geq \lambda \text { and } f^{-1}\left(f\left(A^{U}\right)\right)(x) \geq \mu \\
& \Leftrightarrow A^{L}(x) \geq \lambda \text { and } A^{U}(x) \geq \mu \text { (by Result 4.B) } \\
& \Leftrightarrow x \in A^{[\lambda, \mu]} \\
& \left.\Leftrightarrow f(x) \in f\left(A^{[\lambda, \mu]}\right) \text { (Since Kerf } \subset A^{[\lambda, \mu]}\right) .
\end{aligned}
$$

So $[f(A)]^{\lambda, \mu]}=f\left(A^{[\lambda, \mu]}\right)$. By Proposition 5.5, $R / A \cong R / A^{[\lambda, \mu]}$ and $R^{\prime} / f(A) \cong R /[f(A)]^{[\lambda, \mu]}$. Hence $R / A \cong R^{\prime} / f(A)$. This completes the proof.

\section{References}

[1] R.Biswas,Rosenfeld's fuzzy subgroups with interval-valued membership functions, Fuzzy set and systems 63(1995) 87-90.

[2] J.Y.Choi, S.R.Kim and K.Hur, Interval-valued smooth topological spaces, Honam Math.J.32[4](2010), 711-738.

[3] M.B.Gorzalczany, A method of inference in approximate reasoning based on interval-values fuzzy, sets, Fuzzy sets and Systems 21(1987) 1-17.

[4] K. Hur, J.G.Lee and J.Y.Choi, Interval-valued fuzzy relations, J.Korean Institute of Intelligent systems 19(3)(2009)425-432

[5] H.W.Kang and K.Hur, Interval-valued fuzzy subgroups and rings, Honam. Math. J. 32.(4)(2010), 593-617.

[6] T.K.Mondal and S.K.Samanta,Topology of interval-valued fuzzy sets, Indian J.Pure Appl.Math.30(1)(1999) 20-38.

[7] L.A.Zadeh, Fuzzy sets, Inform. and Control 8(1965),338-353. K. Hur, H.W.Kang and H.K.Song,Intuitionistic fuzzy subgroups and subrings, Honam Math.J.25(1)(2003),19-41. 
[8] — The concept of a linguistic variable and its application to approximate reasoning I, Inform.Sci 8(1975) 199-249.

Hee Won Kang

Department of Mathematics Education, Woosuk University,

Wanju-kun 570-749, Korea.

E-mail:khwon@woosuk.ac.kr 